\title{
Release of extracellular vesicles containing small RNAs from the eggs of Schistosoma japonicum
}

\author{
Shanli Zhu', Sai Wang ${ }^{1}$, Yu Lin ${ }^{1}$, Pengyue Jiang ${ }^{1}$, Xiaobin Cui ${ }^{1}$, Xinye Wang ${ }^{1}$, Yuanbin Zhang ${ }^{1}$ and Weiqing Pan ${ }^{1,2^{*}}$
}

\begin{abstract}
Background: Schistosoma japonicum is a parasitic flatworm that causes human schistosomiasis. Secreted extracellular vesicles (EVs) play a key role in pathogen-host interfaces. Previous studies have shown that $S$. japonicum adult worms can release microRNA (miRNA)-containing EVs, which can transfer their cargo to mammalian cells and regulate gene expression in recipient cells. Tissue-trapped eggs are generally considered the major contributor to the severe pathology of schistosomiasis; however, the interactions between the host and parasite in this critical stage remain largely unknown.

Methods: The culture medium for S. japonicum eggs in vitro was used to isolate EVs. Transmission electron microscopy (TEM) analysis was used to confirm that vesicles produced by the eggs were EVs based on size and morphology. Total RNA extracted from EVs was analyzed by Solexa technology to determine the miRNA profile. The in vitro internalization of the EVs by mammalian cells was analyzed by confocal microscopy. The presence of EVs associated miRNAs in the primary hepatocytes of infected mice was determined by quantitative real-time PCR (qRT-PCR).
\end{abstract}

Results: EVs were isolated from the culture medium of in vitro cultivated S. japonicum eggs. TEM analysis confirmed that nanosized vesicles were present in the culture medium. RNA-seq analysis showed that the egg-derived EVs contained small non-coding RNA (sncRNA) populations including miRNAs, suggesting a potential role in host manipulation. This study further showed that Hepa1-6, a murine liver cell line, internalized the purified EVs and their cargo miRNAs that were detectable in the primary hepatocytes of mice infected with S. japonicum.

Conclusions: Schistosoma japonicum eggs can release miRNA-containing EVs, and the EVs can transfer their cargo to recipient cells in vitro. These results demonstrate the regulatory potential of S. japonicum egg EVs at the parasite-host interface.

Keywords: Schistosoma japonicum, Eggs, Extracellular vesicles, Small non-coding RNAs, miRNAs

\section{Background}

Schistosomiasis is a serious parasitic disease throughout the world's tropical regions, affecting more than 230 million people according to conservative estimates [1]. Three main species of schistosomes infect humans, including Schistosoma haematobium, Schistosoma mansoni and Schistosoma japonicum. In China, S. japonicum remains a major public health problem, although considerable efforts have been devoted to controlling its

\footnotetext{
* Correspondence: wapan0912@aliyun.com

${ }^{1}$ Institute for Infectious Diseases and Vaccine Development, Tongji University School of Medicine, Shanghai, China

${ }^{2}$ Department of Tropical Infectious Diseases, Second Military Medical University, Shanghai, China
}

transmission over the last few decades [2-4]. The complex life-cycle of schistosomes involves multiple developmental stages, including the egg, miracidium, cercaria, schistosomulum and adult worm. Tissue-trapped eggs are generally considered the major contributor to the severe pathology of schistosomiasis, because the parasite has developed strategies to both facilitate the establishment of infection and evade or manipulate the host immune response. Recent investigations have made some progress in the analysis of parasite-host interactions [5-8]. However, a deeper understanding of mechanisms of parasitism is urgently required for discovering novel interventions to control the disease. 
Exosomes are a subtype of small $(30-150 \mathrm{~nm}$ in diameter), membrane-enclosed vesicles, released by various types of mammalian cells in both normal and pathological conditions [9-11]. These vesicles originate from the inward budding of endosomal membranes, which creates multivesicular bodies that fuse with the plasma membrane to effect release [12, 13]. Originally assumed to be cellular waste products, exosomes are now considered highly bioactive extracellular vesicles (EVs) that facilitate intracellular communication [14]. The cargo of exosomes is complex and variable, containing bioactive proteins, functional mRNAs, miRNAs and other small non-coding RNA (sncRNA) species $[9,10,15]$. Therefore, these vesicles can "horizontally" transfer signals to neighboring cells and thus serve as mediators of intercellular communication [15-20]. The discovery of EVs in plants, fungi and bacteria drove the theory that exosomemediated communication could be operated on a crossspecies basis [21-23]. Recent studies of Heligmosomoides polygyrus, Echinostoma caproni, Fasciola hepatica, Dicrocoelium dendriticum, Brugia malayi and Leishmania donovani have demonstrated that EVs are secreted from parasites and may be taken up by host cells [24-29]. The vesicles produced by parasites can deliver virulence factors (proteins and non-coding RNAs) to the host, thus favoring pathogen survival and disease progression [24, 27, 29]. In addition, characterization of EVs released by $S$. mansoni schistosomula [30], S. mansoni adult worms [31] and $S$. japonicum adult worms $[32,33]$ suggested that EVs and their cargo could play a key role in parasite-host interplay. Based upon the increasing realization that EVs facilitate intercellular communication in eukaryotes, we speculate that they contribute to maintenance of the long-term host-parasite interactions during schistosomiasis.

Here, we show that the egg of S. japonicum releases EVs whose size and morphology are consistent with exosomes. These vesicles contain small RNAs, including parasite-specific miRNAs and conserved miRNAs that contain identical seed sites between the host and S. japonicum. Further studies showed that the secreted EVs are internalized by mammalian cells and their cargo miRNAs can be transferred to the recipient cells. Moreover, the egg EVs associated-miRNAs can be detected in the primary hepatocytes of mice infected with $S$. japonicum. These results suggest the regulatory potential of $S$. japonicum egg EVs at the parasite-host interface.

\section{Methods}

\section{Schistosoma japonicum egg isolation, culture and culture} medium collection

For collection of S. japonicum egg secretion products, New Zealand rabbits were percutaneously infected with approximately $1,200 \mathrm{~S}$. japonicum cercariae that were shed from lab-infected snails (Oncomelania hupensis) obtained from the National Institute of Parasitic Disease, Chinese Center for Disease Control and Prevention. Eggs were isolated in sterile conditions from the livers of infected rabbits 6-7 weeks post-infection using the sieving and enzymatic method described by Cai et al. [34]. After collection, the freshly isolated eggs were thoroughly washed three times with PBS (pH 7.4) and then maintained in preheated RPMI-1640 culture medium (Life Technologies, Carlsbad, CA, USA) containing $100 \mathrm{U}$ of penicillin, $100 \mu \mathrm{g} / \mathrm{ml}$ of streptomycin and $0.25 \mu \mathrm{g} / \mathrm{ml}$ amphotericin B (Life Technologies) at $37{ }^{\circ} \mathrm{C}$ under $5 \% \mathrm{CO}_{2}$ at a density of $1 \times 10^{4}$ eggs $/ \mathrm{ml}$ for $24 \mathrm{~h}$. Following $24 \mathrm{~h}$ incubation, eggs and pellets were removed by centrifugation at $3,000 \times g$ at $4{ }^{\circ} \mathrm{C}$. The culture medium was collected for further exosome isolation as described below.

\section{Exosome-like vesicle isolation}

An ExoQuick-TC Exosome Precipitation Kit from System Bioscience (SBI, Mountain View, CA, USA) was used for exosome isolation according to the supplier's protocols. In brief, the medium treated as described above was initially centrifuged at $3,000 \times g$ for $15 \mathrm{~min}$ to remove remaining eggs and cell debris. Exosome isolation reagents ( 0.2 volumes) were added to the supernatants and incubated at $4{ }^{\circ} \mathrm{C}$ overnight. The ExoQuick-TC/medium mixture was then centrifuged at $1,500 \times g$ for $30 \mathrm{~min}$ at room temperature. The supernatants were discarded and pellets were resuspended in $50 \mu \mathrm{l}$ PBS and stored at $-80^{\circ} \mathrm{C}$ until further analysis.

\section{Transmission electron microscopy}

For visualization of the vesicles, purified EVs were applied to 200 mesh formvar-coated EM grids (Agar Scientific, Essex, UK), incubated for $1 \mathrm{~min}$ at room temperature, and then excess sample was removed. The grids were stained with $1 \%$ uranyl acetate (SBI) for $5 \mathrm{~min}$. The grids were then loaded onto the sample holder of the JEM-1230 TEM (JEOL,Tokyo, Japan) and exposed to an $80 \mathrm{kV}$ electron beam for image capture.

\section{Small RNA library preparation and analysis}

For detection of RNA species, total RNA was extracted from EVs released by parasite eggs using Trizol (Life Technologies), and RNA quality was evaluated with an Agilent 2100 system (Agilent Technologies, Santa Clara, CA, USA). The small RNAs between 18 and 30 nt were isolated by $15 \%$ polyacrylamide gel electrophoresis (PAGE) and then ligated into Illumina's proprietary $5^{\prime}$ and $3^{\prime}$ adaptors, and further converted into single strand cDNA. The cDNA products were amplified using a 3 '-adaptor reverse primer and $5^{\prime}$-adaptor forward primer (Illumina, San Diego, CA, USA). The purified PCR products were used for clustering and sequencing by an Illumina Genome Analyzer at the Beijing Genomics Institute, Shenzhen. 
All unique sequences along with their associated read counts were determined from the raw data. First, low quality reads, 3'-adaptor null reads, insert null reads, 5'adaptor contaminants and reads with polyA tails were filtered. The clean datasets were mapped to the draft $S$. japonicum genome sequences (sjr2_scaffold.fasta, downloaded from ftp://lifecenter.sgst.cn:2121/nucleotide/corenucleotide) using the Short Oligonucleotide Alignment Program (http://soap.genomics.org.cn). We investigated the length distribution of the perfectly matched small RNA reads in the libraries. By comparing our sequences with the noncoding RNAs collected in Rfam (Version 11.0) and the NCBI GenBank database, sequenced small RNAs were annotated to different categories, including rRNAs, small nuclear RNAs (snRNAs), repeat associated small RNAs, tRNAs and miRNAs. For miRNA analysis, the unmatched small RNAs were further analyzed against miRbase (version 21) and GenBank to identify known mature miRNAs. Finally, unannotated small RNAs were used for novel miRNA prediction analysis using Mireap (http://sourceforge.net/projects/mireap). RNAfold was used to predict hairpin-like structures.

\section{Quantitative real-time PCR (qRT-PCR) analysis of S. japoni- cum egg EV associated miRNAs}

Stem-loop qRT-PCR was used to validate the presence of miRNAs in S. japonicum egg EVs. Total RNA of egg EVs, culture medium and residuum supernatants (collected after EV isolation) were extracted using Trizol. qRT-PCR analysis was performed as previously described [35]. Briefly, a stem-loop RT primer was used to reversetranscribe mature miRNA to cDNA; the $10 \mu \mathrm{l}$ reverse transcription reactions contained $150 \mathrm{ng}$ of total RNA, 50 $\mathrm{nM}$ of each individual stem-loop RT primer, $0.5 \mathrm{mM}$ dNTP (Takara, Dalian, China), 5 U M-MLV reverse transcriptase (Takara), and $2 \mathrm{U}$ RNase inhibitor. The temperature program was $60 \mathrm{~min}$ at $42^{\circ} \mathrm{C}, 15 \mathrm{~min}$ at $70^{\circ}$ $\mathrm{C}$ and then holding at $4{ }^{\circ} \mathrm{C}$. qRT-PCR was performed using an Applied Biosystems (ABI, Carlsbad, CA, USA) 7500 Sequence Detection system. The $20 \mu \mathrm{l}$ PCR reaction included $2 \mu \mathrm{l}$ of RT product (1:1 dilution), $1 \times$ SYBR Premix Ex Taq (Takara), $0.5 \mu \mathrm{M}$ specific forward primer, and $0.5 \mu \mathrm{M}$ common reverse primer. The reactions were performed at $95^{\circ} \mathrm{C}$ for $30 \mathrm{~s}$, followed by 40 cycles of $95^{\circ} \mathrm{C}$ for $5 \mathrm{~s}$ and $60^{\circ} \mathrm{C}$ for $34 \mathrm{~s}$. Cel-miR-39 was used as an internal control, and the relative miRNAs level was calculated by the $2^{-\Delta \Delta C t}$ method [36]. All reactions were run in triplicate. All primers used are listed in Table 1.

\section{Confocal analysis of S. japonicum egg EV uptake by murine liver cells}

Murine liver Hepa1-6 cells were obtained from the ATCC (CRL-1830) and grown according to the standard protocol in Dulbecco's modified Eagle's medium
Table 1 Sequences of primers used for qRT-PCR

\begin{tabular}{|c|c|c|}
\hline Gene & Name & Sequence $\left(5^{\prime}-3^{\prime}\right)$ \\
\hline \multirow[t]{2}{*}{ sja-miR-71b } & RT stem-loop primer & $\begin{array}{l}\text { CTCAACTGGTGTCGTGGAGTCGG } \\
\text { CAATTCAGTTGAGCGTCTCA }\end{array}$ \\
\hline & Forward primer & $\begin{array}{l}\text { ACACTCCAGCTGGGTGAAAGA } \\
\text { CTTGAGT }\end{array}$ \\
\hline \multirow[t]{2}{*}{ sja-bantam } & RT stem-loop primer & $\begin{array}{l}\text { CTCAACTGGTGTCGTGGAGTCGG } \\
\text { CAATTCAGTTGAGACCAGCT }\end{array}$ \\
\hline & Forward primer & $\begin{array}{l}\text { ACACTCCAGCTGGGTGAGATCG } \\
\text { CGATTA }\end{array}$ \\
\hline \multirow[t]{3}{*}{ cel-miR-39 } & RT stem-loop primer & $\begin{array}{l}\text { CTCAACTGGTGTCGTGGAGTCGG } \\
\text { CAATTCAGTTGAGCAAGCTG }\end{array}$ \\
\hline & Forward primer & $\begin{array}{l}\text { ACACTCCAGCTGGGTGTCACCGG } \\
\text { GTGTAAAT }\end{array}$ \\
\hline & Common reverse primer & CTGGTGTCGTGGAGTCGGCAA \\
\hline \multirow[t]{2}{*}{ mmu-U6 } & Forward primer & $\begin{array}{l}\text { GCTTCGGCAGCACATATACTAA } \\
\text { AAT }\end{array}$ \\
\hline & Reverse primer & CGCTTCACGAATTTGCGTGTCAT \\
\hline
\end{tabular}

(DMEM) (Life Technologies) supplemented with $10 \%$ fetal bovine serum (Life Technologies). Hepa1-6 cells were seeded in 12 -well plates $\left(1 \times 10^{5}\right.$ cells/well $)$ using advanced serum-free DMEM (Life Technologies) for 4 h. Purified EVs from S. japonicum eggs or Hepa1-6 cells were labeled with the green fluorescent dye PKH67 (Sigma-Aldrich, St. Louis., MO, USA) as described by Hazan-Halevy et al. [37] with minor modifications. Briefly, $10 \mu \mathrm{g}$ of the PKH67-stained EVs were washed three times using a 300-kDa Amicon (Merck Millipore, Merck KGaA, Darmstadt, Germany) to remove excess dye, EVs were then added to the cells and incubated for $1 \mathrm{~h}$ at $37^{\circ} \mathrm{C}$. As a control for non-specific labeling of cells, PBS was stained with PKH67, washed, and added to the cells. Following $1 \mathrm{~h}$ incubation, the medium was aspirated, cells were washed twice with PBS, fixed with $4 \%$ formaldehyde solution for $15 \mathrm{~min}$, and washed twice more with PBS; nuclei were stained with 4',6-diamidino-2-phenylindole (DAPI) (Life Technologies). Finally, the cells were observed using confocal fluorescence microscopy (Leica TCS SP5 II, Heidelberg, Germany).

\section{qRT-PCR analysis of Schistosoma-specific miRNAs}

Hepa1-6 cells were incubated with EVs $(10 \mu \mathrm{g}$ total protein per well) derived from $S$. japonicum eggs or Hepa16 cell culture medium (negative control) for $20 \mathrm{~h}$. After washing twice with PBS, the cells were then incubated with $0.25 \%$ trypsin/EDTA (Life Technologies) for $3 \mathrm{~min}$ to eliminate non-specifically adhered EVs. Total RNA was extracted from the incubated cells using Trizol, and miRNAs were reverse transcribed into cDNA using Reverse Transcriptase M-MLV (Takara) as described above. Real-time PCR was performed to assess the Schistosoma-specific miRNAs in the treated Hepa1-6 
cells. Cel-miR-39 was used as an internal control, and the relative miRNAs level was calculated by the $2^{-\Delta \Delta C t}$ method.

\section{Isolation of primary mouse hepatocytes}

To ascertain whether parasite-specific miRNAs were present in the primary hepatocytes of mice infected with S. japonicum, primary mouse hepatocytes were isolated by a two-step collagenase perfusion procedure, as described by $\mathrm{He}$ et al. [38] with minor modifications. Briefly, 6week-old male C57BL/6 mice were purchased from the experimental animal center of the Second Military Medicine University and exposed percutaneously to $20 \mathrm{~S}$. japonicum cercariae. Livers of $S$. japonicum-infected mice (49 days post-infection (dpi) and $80 \mathrm{dpi}, n=5$, respectively) and uninfected mice were initially in situ digested with $0.04 \%$ collagenase type IV and then further digested with $0.08 \%$ collagenase type IV at $37^{\circ} \mathrm{C}$ in a shaking bath for $30 \mathrm{~min}$. The single cell suspensions were harvested by filtration through 400-mesh sieves for removal of the remaining tissue debris and parasite eggs. Next, hepatocytes were isolated by centrifugation of the resulting cell suspensions at $50 \times g$ for $4 \mathrm{~min}$ and further purified by centrifugation at $20 \times g$ for $4 \mathrm{~min}$. Purified hepatocytes were resuspended in DMEM containing $20 \mu \mathrm{g} / \mathrm{ml}$ Ribonuclease A (Sigma-Aldrich) at $37{ }^{\circ} \mathrm{C}$ in a bath for $30 \mathrm{~min}$ to eliminate the miRNA released by schistosome eggs. After three times washing with PBS, the cell pellet was used immediately for extraction of total RNA or stored in liquid nitrogen until further analysis.

\section{qRT-PCR analysis of S. japonicum miRNAs in primary hepatocytes of infected mice}

Total RNA of primary hepatocytes derived from infected and uninfected mice were extracted using Trizol, and parasite miRNAs were reverse transcribed into cDNA using reverse transcriptase M-MLV as described above. qRT-PCR analysis was performed to determine the presence of parasite-specific miRNAs in the primary hepatocytes of infected mice. The U6 gene was used as an internal control. The $2^{-\Delta \mathrm{Ct}}$ method was used to calculate the level of miRNAs.

\section{Statistical analysis}

Results were analyzed using SPSS software (version 16) and statistical analyses were performed using one-way ANOVA. A value of $P<0.05$ was considered statistically significant. Data are expressed as mean \pm standard deviation.

\section{Results}

Isolation and purification of schistosomal eggs

Rapid isolation of viable schistosomal eggs from host hepatic tissue in sterile conditions was a critical step for obtaining EVs. In this study, eggs were isolated and purified from infected rabbits using the sieving and enzymatic methodology described by Cai et al. [34]. The purified egg samples were examined under a light microscope, and we observed that most of the eggs contained a developing embryonic larva (Additional file 1: Figure S1).

\section{EVs isolation from schistosomal eggs and small RNA analysis}

The schistosomal eggs were incubated for $24 \mathrm{~h}$ in standard culture conditions, and vesicles were purified from the culture medium and evaluated by TEM. TEM images showed that eggs cultivated in vitro secreted abundant 30-100 $\mathrm{nm}$ microvesicles consistent with the morphology previously described for $S$. japonicum adult worms [33], confirming that schistosomal eggs also release EVs (Fig. 1).

SncRNAs carried by EVs function as messengers for intercellular communication. Therefore, we identified the small RNA populations associated with schistosomal egg EVs by Solexa deep sequencing. As shown in Fig. 2a, $b$, the length distribution of schistosomal egg EVassociated small RNAs ranged from 18 to 30 nt. rRNAs, snRNAs and repeat associated small RNAs were the dominant classes of small RNAs, followed by intron_sense, tRNAs and miRNAs (Fig. 2c). We found 13 known S. japonicum miRNAs (reads >100) present in the schistosomal egg EV libraries (Table 2 and Additional file 2: Table S1), including three miRNAs (miR-10, bantam and miR-3479-3p) that were present in the plasma of $S$. japonicum infected host rabbits in a previous study [39]. In addition, one novel miRNA was predicted using Mireap (Table 2 and Additional file 2: Table S1). Then, stem-loop qRT-PCR was performed to verify the presence of Sja-bantam and Sja-miR-71b in the RNA isolated from schistosomal egg EVs (Fig. 2d). These findings demonstrated that schistosomal eggs release EVs during development in vitro and these 30-100 nm sized vesicles carry miRNAs that are both parasitespecific and homologs of mammalian (host) (e.g. mouse miR-10) miRNAs.

\section{Uptake of schistosomal egg EVs by murine cells}

To ascertain whether the schistosomal egg-derived EVs can enter mammalian cells, internalization was examined in mouse liver cells. EVs originating from schistosomal eggs or Hepa1-6 cells (positive control) were labeled with the green fluorescent lipid dye PHK67 and incubated with Hepa1-6 cells in vitro. Confocal image analysis revealed efficient internalization of the parasite egg-EVs by murine Hepa1-6 cells (Fig. 3a). qRT-PCR analysis of the treated cells demonstrated that schistosomal egg EVs associated miRNAs (bantam and miR-71b) were detectable in Hepa1-6 cells after $20 \mathrm{~h}$ of incubation 


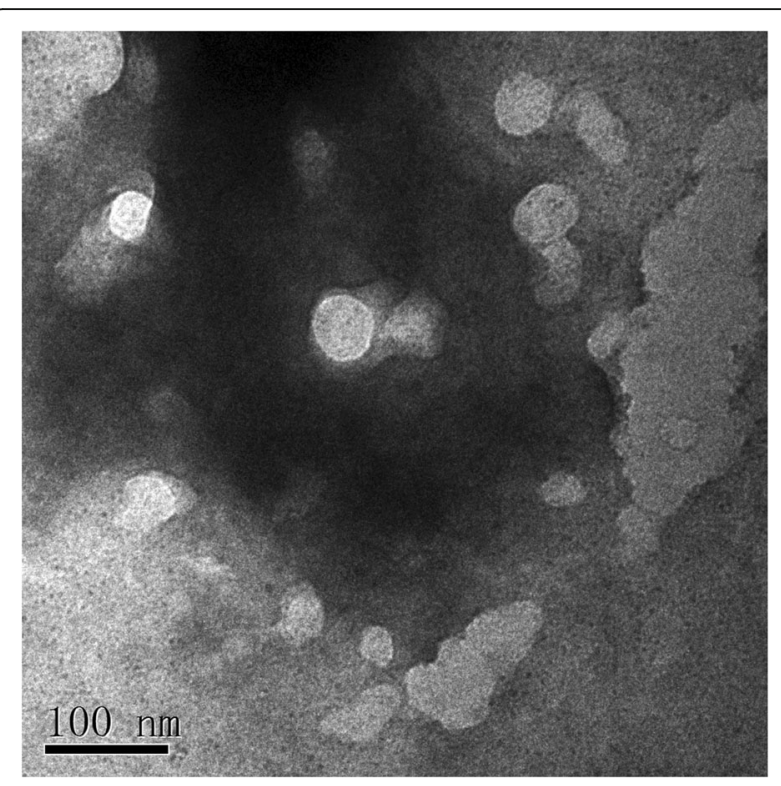

Fig. 1 Characterization of extracellular vesicles (EVs) obtained from the eggs of S. japonicum by transmission electron microscopy (TEM). EVs were isolated from in vitro culture supernatants of S. japonicum eggs and analyzed by TEM at 200,000× magnification. Scale-bar: $100 \mathrm{~nm}$

with parasite EVs (Fig. 3b). The above indicated that the miRNAs carried by schistosomal egg EVs can be transferred to recipient mammalian cells.

\section{qRT-PCR analysis of S. japonicum miRNAs in the primary hepatocytes of infected mice}

To ascertain whether parasite-specific miRNAs were present in the primary hepatocytes of mice infected with $S$. japonicum, primary hepatocytes of infected mice were isolated and detected for two miRNAs that are associated with S. japonicum egg EVs (i.e. Sja-miR-71b and Sja-bantam). The result showed the two miRNAs were detectable in the primary hepatocytes at 49 dpi and 80 dpi (Fig. 4), indicating that parasite-derived miRNAs were present in the primary hepatocytes of infected mice after numerous eggs deposited in the liver.

\section{Discussion}

Extracellular vesicles (EVs), and particularly exosomes, are emerging as one of the major mediators of intercellular communication within mammalian systems. EVs deliver their cargo molecules to specific cells and the transfer of molecules may modulate cellular activity and pathways in recipient cells. Recent studies revealed that helminth parasites such as $B$. malayi, nematode $H$. polygyrus adults, S. mansoni schistosomula and S. japonicum adults, can also release EVs enriched in proteins and non-coding RNAs [24, 27, 30, 33, 40]. However, it remained unknown whether $S$. japonicum eggs, the major agents causing the severe pathology of schistosomiasis, could release EVs. Here, we isolated and cultivated the tissue-trapped eggs of $S$. japonicum, and verified that in vitro cultivated eggs released nanosized vesicles into the culture supernatant (Fig. 1). These nanovesicles potentially represent novel modulators of host and parasite interactions, therefore, their sncRNA content was explored further.

RNA deep sequencing revealed that miRNAs were among the small RNAs in the schistosomal egg EV libraries. Considering RNAs with > 100 reads, 13 previously characterized miRNAs and one potential novel miRNA were identified. Among the 13 known SjamiRNAs identified in the egg EVs, Sja-bantam, Sja-miR10 and Sja-miR-3479-3p were all previously detected in serum obtained from rabbits infected with $S$. japonicum [39]. Also, it has been shown that Sja-miR-277 and SjamiR-3479-3p were detectable in the serum samples of infected mice [41]. We speculate that the circulating miRNAs are protected from degradation by encapsulation in the EVs. Interestingly, bantam and miR-10 were significantly enriched in the libraries of EVs derived from schistosomal adult worms, whereas miR-3479-3p did not appear in those EV libraries [33]. Moreover, it has been shown that five miRNAs (miR-71, miR-71b, miR-1, miR36 , and miR-124) are the most abundant in the egg stage of S. japonicum [34], implying that these miRNAs play important roles in embryo development. In the present study, four of these miRNAs (all except Sja-miR-1) were incorporated into the egg EVs. A similar phenomenon was also observed in the adult stage of S. japonicum [33]. These findings support the notion that miRNAs are selectively packaged into exosomes and delivered to specific cells, although it remains unclear whether the sorting mechanism is related to association with the RNAinduced silencing complex components or the target mRNAs [42-44].

In mammals, cell-to-cell communication could be mediated by EVs, which can deliver their cargo miRNAs to recipient cells $[13,14]$. Exosomal miRNAs have specific functions such as immune response activation and cell development $[45,46]$. The helminthic nematode $H$. polygyrus utilizes exosomal vesicles to increase virulence in a fashion similar to that of the mammalian miRNA transport mechanism [24]. H. polygyrus secretes miRNAloaded vesicles that are accompanied by a nematode AGO protein, most likely to stabilize the miRNAs. Remarkably, $H$. polygyrus vesicles are internalized by mouse cells, which results in suppression of host immunity. Some $H$. polygyrus miRNAs were shown to target in vitro host mRNAs that are related to host immunity. In addition, previous study has shown that 


\begin{tabular}{lr} 
a & \\
\hline Total reads & \multicolumn{1}{c}{ Count } \\
High quality reads & 11842555 \\
3'adaptor null & $11830382(100 \%)$ \\
Insert null & $74055(0.63 \%)$ \\
5'adaptor contaminants & $1871(0.02 \%)$ \\
Small than 18 nt & $14143(0.12 \%)$ \\
Poly A & $237820(2.01 \%)$ \\
Clean reads & $72(0.00 \%)$ \\
\hline
\end{tabular}

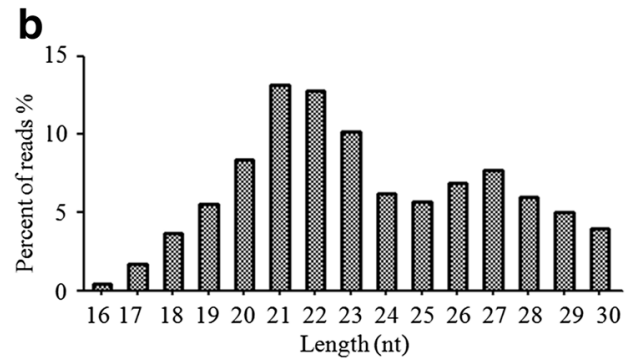

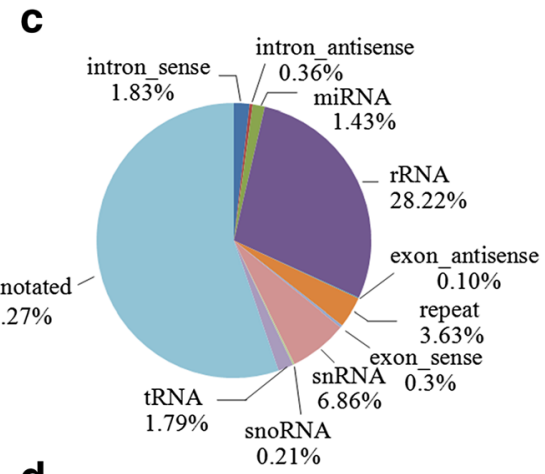

d

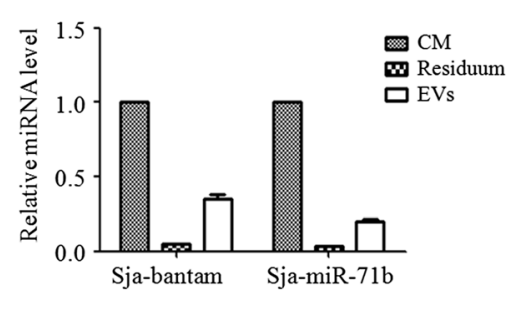

Fig. 2 Identification of small RNAs associated with S. japonicum egg extracellular vesicles (EVs). a Summary of the output of the Solexa data; the percentage in parentheses indicates the percentage of high-quality reads. $\mathbf{b}$ The length distribution of small RNA tags. $\mathbf{c}$ Classification of the small RNAs by comparison with the S. japonicum genome. $\mathbf{d}$ qRT-PCR validation of the abundance of Sja-bantam and Sja-miR-71b in the RNA isolated from S. japonicum egg EVs. Values are normalized to culture medium, based on equal volumes of starting material. Abbreviations: CM, culture medium; EVs, extracellular vesicles; Residuum, supernatant medium from the final step of EV extraction

exogenous plant miRNAs are not only present in serum and tissues of hosts after oral uptake, but they can also regulate the expression of specific genes in the host liver [21]. These findings support the notion of miRNAmediated cross-kingdom regulation, although the mechanisms responsible for exogenous miRNA transfer and function remain largely unknown. In the present study, we showed that murine liver cells could internalize schistosomal egg EVs and their cargo miRNAs in vitro. The results were consistent with previous findings that EVs derived from nematodes and S. japonicum adults could be taken up by mouse small intestinal epithelial cells and liver cells, respectively $[24,33]$. Therefore, EVs derived from $S$. japonicum eggs potentially act as vehicles to package and deliver miRNAs to host cells and regulate host gene expression, which may facilitate parasitism.

Table 2 List of identified miRNAs associated with S. japonicum egg EVs

\begin{tabular}{llllllll}
\hline Small RNA ID & Location & & & Sequence & Reads $^{a}$ & miRNA $^{2}$ \\
\hline t0000052 & SJC_S000027 & 600247 & 600269 & - & CCACCGGGTAGACATTCATTCGC & 29608 & sja-miR-36-3p \\
t0000207 & SJC_S000052 & 314799 & 314820 & + & AACCCTGTAGACCCGAGTTGG & 6156 & sja-miR-10-3p \\
t0000525 & SJC_S000254 & 288019 & 288040 & + & TGAGATCGCGATTAAAGCTGGT & 2307 & sja-bantam \\
t0000729 & SJC_S000054 & 245452 & 245472 & - & TCACAGCCAGTATTGATGAAC & 1332 & sja-miR-2a-3p \\
t0000925 & SJC_S000054 & 245576 & 245597 & - & TGAAAGACGATGGTAGTGAGAT & 1085 & sja-miR-71a \\
t0001363 & SJC_S000055 & 384663 & 384684 & - & TATTGCACTTACCTTCGCCTTG & 1070 & sja-miR-3479-3p \\
t0001942 & SJC_S000471 & 22294 & 22314 & - & TATTATGCAACGTTCACTCT & 1038 & sja-miR-2162-3p \\
t0001985 & SJC_S000102 & 364277 & 364299 & + & AAAGACTTGAGTAGTGAGACGCT & 746 & sja-miR-71b-3p \\
t0002533 & SJC_S000054 & 245393 & 245413 & - & CGTCTCAAAGGACTGTGAGCC & 585 & sja-miR-2b-3p \\
t0002630 & SJC_S000664 & 24730 & 24752 & + & TGACTAGAAAGTGCACTCACTTC & 570 & sja-miR-61 \\
t0003175 & SJC_S000001 & 925810 & 925830 & - & TAAATGCATTTCTGGCCCGT & 554 & sja-miR-277 \\
t0003502 & SJC_S004031 & 7481 & 7503 & + & TCACAACCTACTTGATTGAGGGG & 238 & sja-miR-307 \\
t0005635 & SJC_S000102 & 364554 & 364577 & + & TATCACAGTCCTGCTTAGGTGACG & 139 & sja-miR-2d-3p \\
t0007134 & SJC_S000110 & 287436 & 287456 & - & GGCCTCGTGGTGTAGCGGTTATC & 105 & novel-miR-7 \\
\hline
\end{tabular}




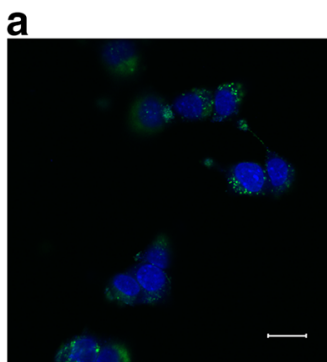

Hepa1-6 EVs-PKH67 b

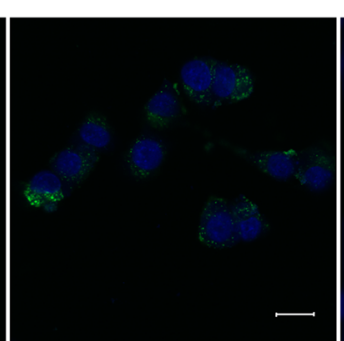

S.japonicum eggs EVs-PKH67

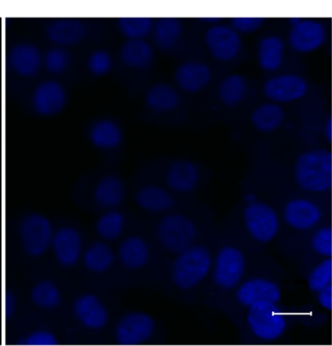

PBS-PKH67

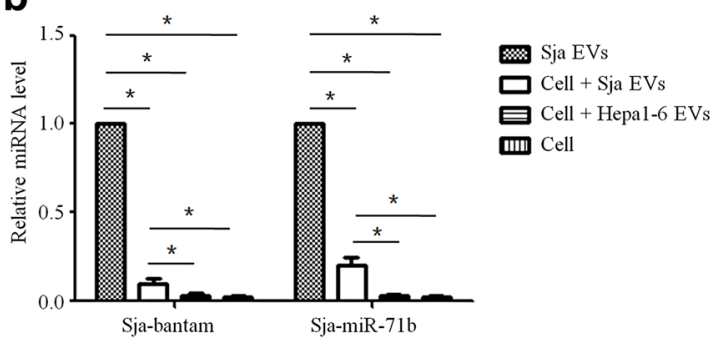

Fig. 3 Schistosoma japonicum egg extracellular vesicles (EVs) and RNAs were internalized by murine liver cells. a Uptake analysis of S. japonicum egg EVs by mouse liver cells detected by confocal microscopy. PKH67-labeled S. japonicum egg EVs, Hepa1-6 EVs, and PHK67-PBS were incubated with Hepa1-6 cells for 1 h. Nuclei were stained with DAPI (blue). Scale-bars: 20 mm. b Relative level of parasite-derived miRNAs (i.e. bantam and miR-71b) in murine liver cells $20 \mathrm{~h}$ post-incubation with $10 \mu \mathrm{g}$ S. japonicum egg EVs following PBS washing. The data were normalized to the level of miRNAs in $10 \mu \mathrm{g}$ of EVs. ${ }^{*} P \leq 0.05$

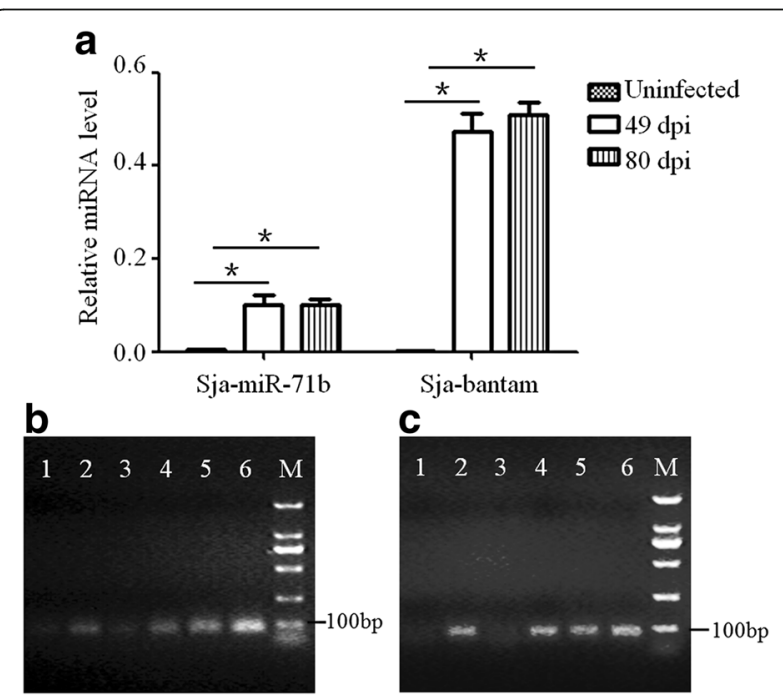

Fig. 4 qRT-PCR analysis of the Sja-miR-71b and Sja-bantam level in primary hepatocytes of infected mice. a qRT-PCR analysis of two of the miRNAs that are associated with S. japonicum egg EVs (i.e. Sja-miR-71b and Sja-bantam) in primary hepatocytes of infected mice at $49 \mathrm{dpi}$ and $80 \mathrm{dpi}$. b, c The PCR products of Sja-miR-71b (68 bp) and Sja-bantam (67 bp). Lanes 1 and 3: primary hepatocytes of uninfected mice at days 49 and 80; Lanes 2 and 4: primary hepatocytes of infected mice at $49 \mathrm{dpi}$ and 80 dpi; Lane 5: S. japonicum egg EVs; Lane 6: S. japonicum eggs
Several lines of evidence have shown that highly conserved miR-71 and bantam are packaged in parasitederived EVs, including from $H$. polygyrus, B. malayi and $S$. mansoni, suggesting that conserved miR-71- and bantamsecretion systems might exist in helminths [24, 27, 30]. In Drosophila, bantam miRNA has been shown to target a tumor-suppress pathway, promoting cellular growth and suppressing cellular apoptosis [47]. Although the role of miR-71 secreted by parasites remains unclear, it has been proposed to be involved in host-pathogen interactions $[24,30]$. In the present study, we observed that SjamiR-71b and Sja-bantam are also incorporated into the EVs derived from schistosomal eggs and these miRNAs can be transferred to murine liver cells via EVs in vitro. More importantly, we found that the parasite-specific miR-71b and bantam were present in the primary hepatocytes of $S$. japonicum infected mice after numerous eggs deposited in the liver.

\section{Conclusions}

Schistosoma japonicum eggs can release miRNAcontaining EVs, which can transfer their cargo miRNAs to recipient cells in vitro. Moreover, egg EVs associated miRNAs (i.e. Sja-miR-71b and Sja-bantam) were detectable in the primary hepatocytes of mice infected S. japonicum. Further work is required to identify the protein components within S. japonicum egg EVs and to understand whether and how each of these cargo molecules contribute to the fundamental interactions 
between the parasite and its host. Further exploration of the functions of these vesicles may help identify novel strategies for control of schistosomiasis.

\section{Additional files}

Additional file 1: Figure S1. S. japonicum eggs isolated from rabbits infected with S. japonicum cercariae $44 \mathrm{dpi}$. (TIF $6650 \mathrm{~kb}$ )

Additional file 2: Table S1. The list of schistosome small RNAs (over 100 reads) associated with $S$. japonicum egg extracellular vesicles (EVs). (XLSX $126 \mathrm{~kb})$

\section{Abbreviations}

DAPI: 4',6-diamidino-2-phenylindole; dpi: days post-infection; EVs: Extracellular vesicles; miRNAs: microRNAs; PAGE: Polyacrylamide gel electrophoresis; qRTPCR: quantitative real-time PCR; sncRNA: small non-coding RNA

TEM: Transmission electron microscopy

\section{Acknowledgements}

We thank the staff of the National Institute of Parasitic Disease, Chinese Center for Disease Control and Prevention for their help with parasite infections.

\section{Funding}

This study was supported by the National Natural Science Foundation of China (grant number 81430051).

\section{Availability of data and materials}

The datasets supporting the conclusions of this article are included within the article and the additional files. The newly-generated miRNA sequence (miR7) was deposited in the GenBank database under the accession number KY021901

\section{Authors' contributions}

SZ and WP conceived and designed the study. SZ, SW, YL, PJ, XC, XW and $Y Z$ performed the experiments and analyzed the data. SZ and WP wrote the manuscript. All authors read and approved the final manuscript.

\section{Competing interests}

The authors declare that they have no competing interests.

\section{Consent for publication}

Not applicable.

\section{Ethics approval and consent to participate}

This study was carried out in strict accordance with the Regulations for the Administration of Affairs Concerning Experimental Animals of the State Science and Technology Commission. All animal studies and protocols were approved by the Internal Review Board of Tongji University School of Medicine (permit number: TJLAC-015-028)

Received: 31 August 2016 Accepted: 12 October 2016

Published online: 08 November 2016

\section{References}

1. Colley DG, Bustinduy AL, Secor WE, King CH. Human schistosomiasis. Lancet. 2014;383:2253-64. doi:10.1016/S0140-6736(13)61949-2.

2. Yang GJ, Sun LP, Hong QB, Zhu HR, Yang K, Gao Q, Zhou XN. Optimizing molluscicide treatment strategies in different control stages of schistosomiasis in the People's Republic of China. Parasit Vectors. 2012;5:260. doi:10.1186/1756-3305-5-260

3. Zhou XN, Wang LY, Chen MG, Wu XH, Jiang QW, Chen XY, et al. The public health significance and control of schistosomiasis in China - then and now. Acta Trop. 2005;96:97-105.

4. Zhou YB, Liang S, Jiang QW. Factors impacting on progress towards elimination of transmission of schistosomiasis japonica in China. Parasit Vectors. 2012:5:275. doi:10.1186/1756-3305-5-275.
5. Zhu L, Liu J, Cheng G. Role of microRNAs in schistosomes and schistosomiasis. Front Cell Infect Microbiol. 2014;4:165. doi:10.3389/fcimb. 2014.00165

6. Driguez P, Mcmanus DP, Gobert GN. Clinical implications of recent findings in schistosome proteomics. Expert Rev Proteomics. 2016;13:19-33. doi:10. 1586/14789450.2016.1116390.

7. Zhu J, Xu Z, Chen X, Zhou S, Zhang W, Chi Y, et al. Parasitic antigens alter macrophage polarization during Schistosoma japonicum infection in mice. Parasit Vectors. 2014;7:122. doi:10.1186/1756-3305-7-122.

8. Zhu L, Dao J, Du X, Li H, Lu K, Liu J, et al. Altered levels of circulating miRNAs are associated Schistosoma japonicum infection in mice. Parasit Vectors. 2015;8:196. doi:10.1186/s13071-015-0806-5.

9. Mathivanan S, Fahner CJ, Reid GE, Simpson RJ. Exocarta 2012: Database of exosomal proteins, RNA and lipids. Nucleic Acids Res. 2012;40:D1241-4. doi: 10.1093/nar/gkr828.

10. Théry C, Zitvogel L, Amigorena S. Exosomes: composition, biogenesis and function. Nat Rev Immunol. 2002:2:569-79.

11. Conde-Vancells J, Rodriguez-Suarez E, Embade N, Gil D, Matthiesen R, Valle $\mathrm{M}$, et al. Characterization and comprehensive proteome profiling of exosomes secreted by Hepatocytes. J Proteome Res. 2008;7:5157-66.

12. Simpson RJ, Lim JW, Moritz RL, Mathivanan S. Exosomes: proteomic insights and diagnostic potential. Expert Rev Proteomics. 2009;6:267-83. doi:10.1586/ epr.09.17.

13. Mittelbrunn M, Sánchez-madrid F. Intercellular communication: diverse structures for exchange of genetic information. Nat Rev Mol Cell Biol. 2012; 13:328-35. doi:10.1038/nrm3335.

14. Théry C. Exosomes: secreted vesicles and intercellular communications. F1000 Biol Rep. 2011;3:15. doi:10.3410/B3-15.

15. Valadi H, Ekström K, Bossios A, Sjöstrand M, Lee JJ, Lötvall JO. Exosomemediated transfer of mRNAs and microRNAs is a novel mechanism of genetic exchange between cells. Nat Cell Biol. 2007:9:654-9.

16. Li J, Liu K, Liu Y, Xu Y, Zhang F, Yang H, et al. Exosomes mediate the cell-tocell transmission of IFN-a-induced antiviral activity. Nat Immunol. 2013;14: 793-803. doi:10.1038/ni.2647

17. Mittelbrunn M, Gutiérrez-Vázquez C, Villarroya-Beltri C, González S, SánchezCabo F, González MÁ, et al. Unidirectional transfer of microRNA-loaded exosomes from T cells to antigen-presenting cells. Nat Commun. 2011;2:282. doi:10.1038/ncomms1285.

18. Montecalvo A, Larregina AT, Shufesky WJ, Stolz DB, Sullivan ML, Karlsson JM et al. Mechanism of transfer of functional microRNAs between mouse dendritic cells via exosomes. Blood. 2012;119:756-66. doi:10.1182/blood2011-02-338004

19. Meckes Jr DG, Shair KH, Marquitz AR, Kung CP, Edwards RH, Raab-Traub N. Human tumor virus utilizes exosomes for intercellular communication. Proc Natl Acad Sci USA. 2010;107:20370-5. doi: 10.1073/pnas.1014194107

20. Pegtel DM, Cosmopoulos K, Thorley-Lawson DA, van Eijndhoven MA Hopmans ES, Lindenberg JL, et al. Functional delivery of viral miRNAs via exosomes. Proc Natl Acad Sci USA. 2010;107:6328-33. doi: 10.1073/pnas. 0914843107

21. Zhang L, Hou D, Chen X, Li D, Zhu L, Zhang Y, et al. Exogenous plant MIR168a specifically targets mammalian LDLRAP1: evidence of crosskingdom regulation by microRNA. Cell Res. 2012;22:107-26.

22. Oliveira DL, Freire-de-Lima CG, Nosanchuk JD, Casadevall A, Rodrigues ML, Nimrichter L. Extracellular vesicles from Cryptococcus neoformans modulate macrophage functions. Infect Immun. 2010;78(4):1601-9. doi:10.1128/IAI 01171-09.

23. Koeppen K, Hampton TH, Jarek M, Scharfe M, Gerber SA, Mielcarz DW, et al. A novel mechanism of host-pathogen interaction through sRNA in bacterial outer membrane vesicles. PLoS Pathog. 2016;12(6):e1005672. doi:10.1371/ journal.ppat.1005672

24. Buck AH, Coakley G, Simbari F, McSorley HJ, Quintana JF, Le Bihan T, et al. Exosomes secreted by nematode parasites transfer small RNAs to mammalian cells and modulate innate immunity. Nat Commun. 2014:5: 5488. doi:10.1038/ncomms6488.

25. Marcilla A, Trelis M, Cortés A, Sotillo J, Cantalapiedra F, Minquez MT, et al. Extracellular vesicles from parasitic helminths contain specific excretory/ secretory proteins and are internalized in intestinal host cells. Plos One. 2012;7:e45974. doi:10.1371/journal.pone.0045974.

26. Bernal D, Trelis M, Montaner S, Cantalapiedra F, Galiano A, Hackenberg M, Marcilla A. Surface analysis of Dicrocoelium dendriticum. The molecular 
characterization of exosomes reveals the presence of miRNAs. J Proteomics. 2014;105:232-41. doi:10.1016/j.jprot.2014.02.012.

27. Zamanian M, Fraser LM, Agbedanu PN, Harischandra H, Moorhead AR, Day $T A$, et al. Release of small RNA-containing exosome-like vesicles from the human filarial parasite Brugia malayi. PLoS Negl Trop Dis. 2015;9:e0004069. doi:10.1371/journal.pntd.0004069.

28. Lambertz U, Oviedo Ovando ME, Vasconcelos EJ, Unrau PJ, Myler PJ, Reiner NE. Small RNAs derived from tRNAs and rRNAs are highly enriched in exosomes from both old and new world Leishmania providing evidence for conserved exosomal RNA Packaging. BMC Genomics. 2015;16:15. doi:10. 1186/s12864-015-1260-7.

29. Silverman JM, Clos J, De'Oliveira CC, Shirvani O, Fang Y, Wang C, et al. An exosome-based secretion pathway is responsible for protein export from Leishmania and communication with macrophages. J Cell Sci. 2010;123:84252. doi:10.1242/jcs.056465.

30. Nowacki FC, Swain MT, Klychnikov Ol, Niazi U, Ivens A, Quintana JF, et al. Protein and small non-coding RNA-enriched extracellular vesicles are released by the pathogenic blood fluke Schistosoma mansoni. J Extracell Vesicles. 2015;4:28665. doi:10.3402/jev.v4.28665.

31. Sotillo J, Pearson M, Potriquet J, Becker L, Pickering D, Mulvenna J, et al. Extracellular vesicles secreted by Schistosoma mansoni contain protein vaccine candidates. Int J Parasitol. 2016;46:1-5. doi:10.1016/j. ijpara.2015.09.002.

32. Wang L, Li Z, Shen J, Liu Z, Liang J, Wu X, et al. Exosome-like vesicles derived by Schistosoma japonicum adult worms mediates M1 type immuneactivity of macrophage. Parasitol Res. 2015;114:1865-73. doi:10.1007/s00436015-4373-7.

33. Zhu L, Liu J, Dao J, Lu K, Li H, Gu H, et al. Molecular characterization of S. japonicum exosome-like vesicles reveals their regulatory roles in parasitehost interactions. Sci Rep. 2016;6:25885. doi:10.1038/srep25885.

34. Cai P, Piao X, Hao L, Liu S, Hou N, Wang H, Chen Q. A deep analysis of the small non-coding RNA population in Schistosoma japonicum eggs. PLoS One. 2013;8:e64003. doi:10.1371/journal.pone.0064003.

35. Xue X, Sun J, Zhang Q, Wang Z, Huang Y, Pan W. Identification and characterization of novel microRNAs from Schistosoma japonicum. Plos One. 2008;3:e4034. doi:10.1371/journal.pone.0004034.

36. Sohn W, Kim J, Kang SH, Yang SR, Cho JY, Cho HC, et al. Serum exosomal microRNAs as novel biomarkers for hepatocellular carcinoma. Exp Mol Med. 2015;47:e184. doi:10.1038/emm.2015.68.

37. Hazan-Halevy I, Rosenblum D, Weinstein S, Bairey O, Raanani P, Peer D. Cellspecific uptake of mantle cell lymphoma-derived exosomes by malignant and non-malignant B-lymphocytes. Cancer Lett. 2015;364:59-69. doi:10. 1016/j.canlet.2015.04.026.

38. He X, Sai X, Chen C, Zhang Y, Xu X, Zhang D, Pan W. Host serum miR-223 is a potential new biomarker for Schistosoma japonicum infection and the response to chemotherapy. Parasit Vectors. 2013;6:272. doi:10.1186/17563305-6-272.

39. Cheng G, Luo R, Hu C, Cao J, Jin Y. Deep sequencing-based identification of pathogen-specific microRNAs in the plasma of rabbits infected with Schistosoma japonicum. Parasitology. 2013;140(14):1751-61. doi:10.1017/ S0031182013000917.

40. Marcilla A, Martin-Jaular L, Trelis M, de Menezes-Neto A, Osuna A, Bernal D, et al. Extracellular vesicles in parasitic diseases. J Extracell Vesicles. 2014;3: 25040. doi:10.3402/jev.v3.25040.

41. Cai P, Gobert GN, You H, Duke M, McManus DP. Circulating miRNAs: potential novel biomarkers for hepatopathology progression and diagnosis of schistosomiasis japonica in two murine models. PLoS Negl Trop Dis. 2015;9:e0003965. doi:10.1371/journal.pntd.0003965.

42. Mitchell PS, Parkin RK, Kroh EM, Fritz BR, Wyman SK, Pogosova-Agadjanyan EL, et al. Circulating microRNAs as stable blood-based markers for cancer detection. Proc Natl Acad Sci USA. 2008;105:10513-8. doi: 10.1073/pnas. 0804549105

43. Villarroya-Beltri C, Gutiérrez-Vázquez C, Sánchez-Cabo F, Pérez-Hernández D, Vázquez J, Martin-Cofreces N, et al. Sumoylated hnRNPA2B1 controls the sorting of miRNAs into exosomes through binding to specific motifs. Nat Commun. 2013;4:2980. doi:10.1038/ncomms3980.

44. Ohshima K, Inoue K, Fujiwara A, Hatakeyama K, Kanto K, Watanabe Y, et al. Let-7 microRNA family is selectively secreted into the extracellular environment via exosomes in a metastatic gastric cancer cell line. Plos One. 2012:5:e13247. doi:10.1371/journal.pone.0013247.
45. Robbins PD, Morelli AE. Regulation of immune responses by extracellular vesicles. Nat Rev Immunol. 2014;14:195-208. doi:10.1038/nri3622.

46. Xiao J, Pan Y, Li XH, Yang XY, Feng YL, Tan HH, et al. Cardiac progenitor cell-derived exosomes prevent cardiomyocytes apoptosis through exosomal miR-21 by targeting PDCD4. Cell Death Dis. 2016;7:e2277. doi:10.1038/cddis. 2016.181.

47. Nolo R, Morrison CM, Tao C, Zhang X, Halder G. The bantam microRNA is a target of the hippo tumor-suppressor pathway. Curr Biol. 2006;16:1895-904.

\section{Submit your next manuscript to BioMed Central and we will help you at every step:}

- We accept pre-submission inquiries

- Our selector tool helps you to find the most relevant journal

- We provide round the clock customer support

- Convenient online submission

- Thorough peer review

- Inclusion in PubMed and all major indexing services

- Maximum visibility for your research

Submit your manuscript at www.biomedcentral.com/submit
Biomed Central 